\title{
Optimization of the design for the LCLS undulator line
}

E. Gluskin, N.A. Vinokurov ${ }^{\dagger}$, R.J. Dejus, P. Emma ${ }^{\ddagger}$, E.R. Moog, H.-D. Nuhn ${ }^{\dagger}$, and I.B.

Vasserman

Advanced Photon Source, Argonne National Laboratory, Argonne, IL 60439, USA

† Budker Institute of Nuclear Physics, 630090 Novosibirsk, Russian Federation

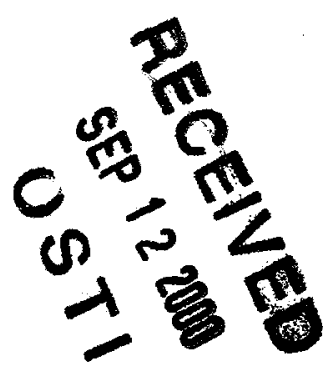

$\ddagger$ Stanford Linear Accelerator Center, Stanford, CA 94309, USA

The Linac Coherent Light Source (LCLS) undulator line will consist of undulator segments separated by breaks of various lengths. Focusing quadrupoles, in a FODO lattice, and electron beam diagnostics will be located in the breaks, and every third break will be longer to also accommodate photon diagnostics. The electron beam beta function and the undulator period were selected to minimize the saturation length. The FEL simulation code RON has been used to optimize parameters such as the length of the undulators and the break lengths between undulators. Different break lengths after the first three undulators have been found to help reduce the overall undulator line saturation length. Tolerances for individual undulators have also been determined.

\section{Introduction}

One possibility for creating an x-ray free-electron laser (FEL) is to use the superradiant, or SASE (self-amplified spontaneous emission), scheme. This scheme involves only two elements: an undulator and the electron beam propagating through the undulator. 


\section{DISCLAIMER}

This report was prepared as an account of work sponsored by an agency of the United States Government. Neither the United States Government nor any agency thereof, nor any of their employees, make any warranty, express or implied, or assumes any legal liability or responsibility for the accuracy, completeness, or usefulness of any information, apparatus, product, or process disclosed, or represents that its use would not infringe privately owned rights. Reference herein to any specific commercial product, process, or service by trade name, trademark, manufacturer, or otherwise does not necessarily constitute or imply its endorsement, recommendation, or favoring by the United States Government or any agency thereof. The views and opinions of authors expressed herein do not necessarily state or reflect those of the United States Government or any agency thereof. 


\section{DISCLAIMER}

Portions of this document may be illegible in electronic image products. Images are produced from the best available original document. 
The electron beam is unstable in that it bunches at the wavelength of the fundamental harmonic of the spontaneous undulator radiation. When the bunching is small, its dependence is linear, so the Fourier harmonics of the beam current at this frequency grow exponentially with distance traveled through the undulator. The gain length is the characteristic length where the squared magnitude of the fundamental Fourier harmonic increases by a factor of $e$. At some distance from the beginning of the undulator, the electron beam has become significantly bunched and there is no further growth; this distance is the saturation length. The coherent undulator radiation produced by the bunched beam is the output of the FEL. An advantage of this FEL scheme is the absence of mirrors, which are a serious problem for $\mathrm{x}-$ ray wavelengths. A disadvantage is that the radiation spectrum is relatively wide and the efficiency is low. From the point of view of building such a device, the main problems are obtaining a high-current low-emittance low-energy-spread electron beam to keep the saturation length within reasonable limits (i.e., not much over 100 meters) and to meet the tight tolerances for field errors, misalignments and steering errors of the undulator.

Typically the saturation length is about 20 times the gain length. For an FEL that is barely (or not) long enough to saturate, nearly all the output light comes from the end of the undulator line. Most of the line is devoted to bunching the electron beam by linearly amplifying the initial particle density fluctuations. Therefore, the goal in optimizing this part of the undulator line is to minimize the gain length.

\section{Optimal period and focusing}

The main parameters of the LCLS project [1] are listed in Table 1 
A challenging feature of the LCLS undulator is the high ratio of 4.5 between the beam emittance and the "minimum radiation emittance" for the light (i.e., wavelength divided by $4 \pi$ ). This means that only a small fraction of the particles will overlap with the light so as to be involved in the radiation amplification. To improve the situation, one could increase the beam energy further. Then (for the same normalized emittance) the beam emittance would decrease. There are two limitations to the increase of energy, however. The first is that there would be an increase in the energy spread due to quantum fluctuations of radiation. The second is that the linac energy for the LCLS is limited. This affects the possible choice for undulator period and beta function.

In Fig. 1, the dependence of the saturation length on the undulator period and matched beta function (lines of equal saturation length) for the planar permanent magnet undulator is shown. This dependence was obtained using the formulas of Halbach [2] and Ming Xie [3] and takes into account both the energy spread due to quantum excitation and the undulator "filling factor" (the fraction of the undulator line length occupied by undulators rather than by the breaks between undulator sections). The wavelength of the output radiation is kept at 1.5 $\AA$, but the magnetic field strength changes in accordance with Halbach's relation [2], and the electron beam energy must change as well to keep the wavelength constant. Fig. 1 shows that the design values of $0.03 \mathrm{~m}$ for the undulator period (corresponding to the $14.35 \mathrm{GeV}$ energy) and $20 \mathrm{~m}$ for the beta function are close to optimal. For lower energy spread and emittance, the optimal undulator period decreases. 
The use of a superconducting helical undulator was also considered. For a period of $0.24 \mathrm{~m}$, a field of $1.3 \mathrm{~T}$, and with other parameters the same as for the planar permanent magnet option, the saturation length is about $70 \mathrm{~m}$. Although this saturation length is shorter than for a planar undulator, there remain some as yet untested aspects to the mechanical design of a superconducting helical device. Since planar permanent magnet undulators are an established technology, they will be used for the LCLS project.

Irregularities and imperfections

The linear theory of high gain is well developed now (see, for example, [4]). Nevertheless, the design of a real magnetic system for a short-wavelength high-gain FEL requires consideration of an inhomogeneous nonsymmetric magnetic system with separated focusing quadrupoles inserted into the breaks between undulator sections. Field, steering, and alignment errors must be considered. The linear time-independent code RON [5] was written for the optimization of such magnetic systems. It was used successfully for the design optimization of the Argonne FEL [6], which first used the separated-focusing approach and has tested and proved many features of the current LCLS design. This code has now also . been used for the optimization of the LCLS undulator line.

The simplest way to provide proper focusing is to use a FODO lattice, and this choice has been made for the LCLS project. The magnetic system of the undulator line will consist of undulator sections with breaks between the undulators where quadrupoles and beam position monitors will be installed. After every third undulator, the break will be longer so that photon diagnostics can be installed as well. This structure is geometrically similar to 
the existing APS FEL except that the photon diagnostics are only after every third undulator. Another lattice based on quadrupole triplets between undulators was considered and rejected because of very tight tolerances for the relative alignment of the three quadrupole centers.

The following parameter choices were made, based on the results of RON calculations:

1. The optimal undulator length was found to be near $3.4 \mathrm{~m}$. For shorter lengths, the "filling factor" is less, making the effective gain length longer. (This assumes that the break length is kept at about $.2 \mathrm{~m}$, which is required by phasing conditions.) For longer undulator lengths, the gain length at a beam energy of $4.5 \mathrm{GeV}^{*}$ increases due to the variation of the beta function within the undulator. The longer lengths are also more difficult mechanically.

2. The optimal average value for the beta function was found to be $20 \mathrm{~m}$. The focal lengths of the quadrupoles will be chosen accordingly.

3. The break lengths between undulators were optimized by calculating the corrections to the "resonance" break length due to the effect of finite emittance and diffraction.

4. An option that included magnetic bunchers between the undulator sections was considered and optimized. No significant improvement was found, so no magnetic bunchers are included in the undulator line design.

* The $4.5 \mathrm{GeV}$ mode is planned for initial FEL commissioning, to produce a wavelength of 15 nm. 
5. The effect of the residual quadrupole misalignment after simulated beam-based alignment [7] was calculated for the optimized undulator line. The increase of the saturation length was found to be about $10 \mathrm{~m}$.

6. The effect of the spread of deflection parameters $K$ in different undulator sections was simulated. This way the corresponding tolerances were found.

Tolerances for the undulator section

The aim of our optimization is to minimize the gain length and consequently the saturation length. There are tens of significant parameters in the system, and a deviation in any of these parameters will increase the gain length. A tolerance budget was worked out for the various parameters so that the overall gain length increase does not exceed $3 \%$, which corresponds to a 4-m increase in saturation length. Tolerances were set assuming simultaneous worst cases for all parameters. The overall tolerances for the undulator line were used to determine tolerances for a single undulator section.

The following requirements for the undulator section field errors were developed.

1. The trajectory walk-out from a straight line must not exceed 2 microns. The beam-based alignment technique will minimize deviations in the transverse beam coordinates near the beam position monitors (BPMs) between the undulator sections, so the trajectory walkouts $x(z)$ and $y(z)$ with zero initial (at the upstream BPM) and final (at the downstream BPM) coordinates have to be specified: $x(z)=\frac{1}{\gamma} \int_{0}^{z} I_{1 x}\left(z^{\prime}\right) d z^{\prime}, y(z)=\frac{1}{\gamma} \int_{0}^{z} I_{1 y}\left(z^{\prime}\right) d z^{\prime}$, where $\gamma$ is the relativistic factor, 


$$
\begin{aligned}
& I_{\mathrm{tx}}(z)=\frac{e}{m c^{2}}\left[\int_{0}^{z} B_{x}\left(z^{\prime}\right) d z^{\prime}-\frac{1}{L} \int_{0}^{L} \int_{0}^{z^{\prime}} B_{x}\left(z^{\prime \prime}\right) d z^{\prime \prime} d z^{\prime}\right], \\
& I_{\mathrm{l} y}(z)=\frac{e}{m c^{2}}\left[\int_{0}^{z} B_{y}\left(z^{\prime}\right) d z^{\prime}-\frac{1}{L} \int_{0}^{L} \int_{0}^{z^{\prime}} B_{y}\left(z^{\prime \prime}\right) d z^{\prime \prime} d z^{\prime}\right],
\end{aligned}
$$

$e$ and $m$ are electron charge and mass, $c$ is the velocity of light, $B_{x}$ and $B_{y}$ are the measured transverse components of magnetic field, and $L$ is the cell length (the distance between $\mathrm{BPMs})$. The 2-micron deviations in both the $\mathrm{x}$ and $\mathrm{y}$ directions gives an increase in the gain length of less than $0.2 \%$, and can be achieved with present magnetic measurement and tuning techniques.

2. The reduction in spectral intensity of the zero-angle radiation must not exceed $4 \%$. The spectral intensity of the zero-angle radiation is $\frac{e^{2} k^{2}|A|^{2}}{2 \pi c \gamma^{2}}$, where $k$ is the fundamental harmonic wavevector of the undulator radiation, and $A=\int_{0}^{L} I_{1 y}(z) e^{-i \frac{k}{2 \gamma^{2}}\left[z+\int_{0}^{2} I_{1 x}^{2}\left(z^{\prime}\right) d z^{\prime}+\int_{0}^{z} l_{1 y}^{2}\left(z^{\prime}\right) d z^{\prime}\right]} d z$

The "reduction" is as compared with an ideal undulator, but in practice the comparison can be with the best undulator, i.e., the one which gives the highest value of $|A|$. A $4 \%$ intensity reduction corresponds to an increase in the gain length by $1.1 \%$.

3. The calculated particle phase deviation from the design value must be less than $10^{\circ}$. This phase is simply the particle-wave slippage: $\varphi=\frac{k}{2 \gamma^{2}}\left[L+\int_{0}^{L} I_{1 x}^{2}(z) d z+\int_{0}^{L} I_{1 y}^{2}(z) d z\right]$, and the 
"design value" is an integer multiple of $2 \pi$. A $10^{\circ}$ dephasing causes an increase in gain length of $1.7 \%$.

4. The undulator median plane must be defined (and after that aligned) with an accuracy better than 50 microns vertically. If the beam is off-axis vertically by 50 microns, the beam will see a stronger undulator field, resulting in about $10^{\circ}$ of additional phase slippage.

\section{Magnetic and mechanical designs}

The magnetic design of an undulator to meet the parameters given in Table 1 has been completed. The undulators will rely on proven hybrid technology, using vanadium permendur poles and Nd-Fe-B permanent magnets. The grade of magnet material selected is one with very high coercivity to increase the magnets' resistance to radiation-induced demagnetization. The poles and magnets will be rectangular rather than wedged, to help keep the mechanical design and fabrication of the magnetic structure more straightforward. With a period length of $30 \mathrm{~mm}$, these undulators will be similar enough to the APS-standard 33-mmperiod undulators that the tuning techniques developed for the APS undulators should transfer directly. In fact, it is encouraging that the tolerances presented in the previous section are already met by the undulators that were tuned magnetically for installation in the APS FEL.

Straightness of the trajectory is a significant requirement for the undulators, and a proper design for the ends of the undulators will help keep the trajectory straight. The strengths of the poles at the ends of the undulators will go in the sequence $0.25,0.75,1$. This 
gives an entrance into (or exit from) the undulator that has no angle kick and no trajectory offset.

Proper phasing between undulators also demands proper tuning of the undulator ends.

The magnetic phasing must match the physical distance between undulators. End phase tuning techniques were developed for the APS FEL that could tune the phasing by $\pm 38^{\circ}$; these techniques will be applied to the LCLS undulators.

The mechanical design for the undulators is in progress.

This work is supported by the U.S. Department of Energy, Office of Basic Energy Sciences, under Contract No. 31-109-Eng-38.

\section{References}

1. LCLS Design Study Report, SLAC-R521 and UC-414, Rev. Dec. 1998.

2. K. Halbach, J. de Physique 44, Colloque C1, supplement to \#2 (1983), C1.

3. M. Xie, IEEE Proceedings of the 1995 Particle Accelerator Conference, (IEEE, 1996), p. 183.

4. K.-J. Kim and M. Xie, Nucl. Instrum. Methods A 331 (1993) 359.

5. R. Dejus et al., Nucl. Instrum. Methods A 429 (1999) 225.

6. E. S. Gluskin et al., Nucl. Instrum. Methods A 429 (1999) 358.

7. P. Emma et al., Nucl. Instrum. Methods A 429 (1999) 407. 
Table 1. Some parameters of the LCLS project.

\begin{tabular}{|l|l|}
\hline Radiation wavelength & $0.15 \mathrm{~nm}$ \\
\hline Beam energy & $14.35 \mathrm{GeV}$ \\
\hline Normalized emittance & $1.5 \mathrm{~mm}-\mathrm{mrad}$ \\
\hline Beam peak current & $3.4 \mathrm{kA}$ \\
\hline Energy spread (standard deviation) & $3 \mathrm{MeV}$ \\
\hline Focusing & FODO \\
\hline Undulator period & $30 \mathrm{~mm}$ \\
\hline Undulator parameter K & 3.71 \\
\hline Undulator effective field & $13.250 \mathrm{kG}$ \\
\hline Nominal magnetic gap & $6 \mathrm{~mm}$ \\
\hline Undulator length & $3.36 \mathrm{~m}$ \\
\hline Break length (short) & $0.231 \mathrm{~m}$ \\
\hline Break length (long) & $0.463 \mathrm{~m}$ \\
\hline Supercell length (6 undulators) & $22.010 \mathrm{~m}$ \\
\hline Number of undulators & 33 \\
\hline
\end{tabular}




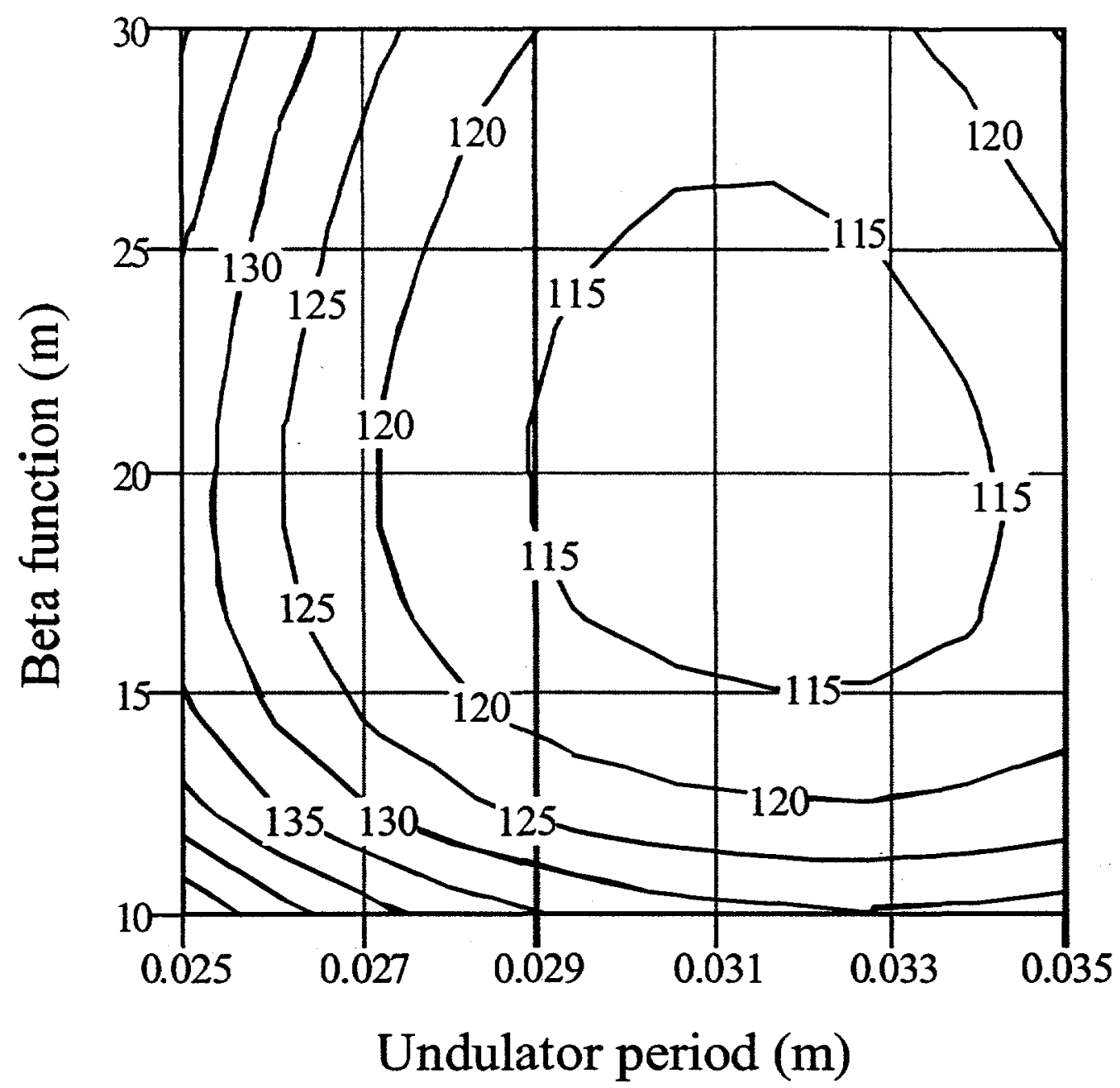

Fig. 1. Contours of constant saturation length. While the wavelength of the light produced is always $1.5 \AA$, the magnetic field strength in the undulator is assumed to change with the undulator period length according to Halbach's formula [2], and the energy of the electron beam changes as needed to preserve the wavelength of the light. The effect of the increase in the electron beam energy spread along the undulator line due to quantum fluctuations is included in the calculation. All numbers are in meters. 